\title{
Investigation of Potential Anticarcinogenic Effects of Corilagin in Lung Cancer Cells
}

\author{
Cagla Rencuzogullari ${ }^{1}$, Zeynep Birsu Cincin ${ }^{1,2}$ (D) Elif Sinem Iplik ${ }^{3}$, Yusuf Baran ${ }^{4}$ (D), Bedia Cakmakoglu ${ }^{1}$ \\ ${ }^{1}$ Istanbul University, Aziz Sancar Institute of Experimental Medicine, Department of Molecular Medicine, Istanbul, Turkey. \\ ${ }^{2}$ Nisantasi University, Faculty of Engineering and Architecture, Department of Genetics and Bioengineering, Istanbul,Turkey. \\ ${ }^{3}$ Istanbul Yeni Yuzyil University, Faculty of Pharmacy, Department of Biochemistry, Istanbul, Turkey. \\ ${ }^{4}$ Izmir Institute of Technology, Faculty of Science, Department of Molecular Biology and Genetics, Izmir, Turkey. \\ Correspondence Author: Bedia Cakmakoglu \\ E-mail: bedia@istanbul.edu.tr \\ Received: $21.03 .2018 \quad$ Accepted: 30.05 .2018
}

\begin{abstract}
Objective: Lung cancer (LC) is the most extensive reason of cancer associated deaths in men and women in the world. LC categorizes into two main groups due to their molecular clinicopathological features and therapeutic responses. Non-small cell lung cancer (NSCLC) is the main subgroup that consists of nearly $85 \%$ of all lung cancer types. Corilagin, a biologically active ellagitannin, could be extracted from Phyllanthus species which are known as Chinese medicinal plant. It has been recently shown that Corilagin could exert anti-inflammatuar and antioxidative effects in different experimental cancer models. However, the molecular effects of Corilagin in NSCLC remain unclear.

Methods: In this study, the antiproliferative and apoptotic effects of Corilagin were identified by WST-1 cell proliferation test, caspase-3 and mitochondrial membrane potential (MMP).

Results: We found that Corilagin significiantly suppressed the proliferation of NSCLC cells. Furthermore, we also showed that Corilagin could contribute apoptosis by inducing activity of caspase-3 molecule and loss of MMP.

Conclusion: Taken together, our study first showed that Corilagin could be a new treatment method for NSCLC after verifying its effects with in vivo and clinical studies.
\end{abstract}

Keywords: Corilagin; antiapoptotic; non-small cell lung cancer

\section{INTRODUCTION}

Lung cancer (LC) is the most extensive reason of cancer associated deaths in men and women in the world (1). LC classifies into two subgroups based on their molecular clinical behaviors and therapeutic responses (2). Non-small cell lung cancer (NSCLC) is the main subgroup that consists of nearly $85 \%$ of all lung cancer types (3). NSCLC is less responsive to chemotherapeutics in contrast to small cell lung cancer (SCLC) (4). Despite of technological developments on surgical and diagnosis methods, the survival rate of NSCLC patients at metastatic stage is still poor (5-8). Therefore, recent studies turn towards the biomarker and drug researches to find effective and non-toxic treatment methods (9-11).

Corilagin involves in biologically active tannin family which could be extracted from Phyllanthus species. (12-15) Previous studies determined that Corilagin could show antihypertensive and anti-atherogenic features in experimental models of cardiovascular diseases (16-18). Furthermore, Corilagin could play an important role as a radical scavenger in superoxide anion system. Regarding to its health-beneficial impacts, researchers started to work about whether Corilagin had anticarcinogenic effects for cancer cells with in vivo and in vitro studies (19-23). However, the effects of Corilagin and its molecular mechanisms on LC remain unclear.

To our knowledge, there is no established study about the antitumor effects in NSCLC cells. Herein, our goal was to understand cellular and molecular effects of Corilagin in NSCLC cells as exploring novel and efficient diagnostic methods.

\section{METHODS}

\subsection{Cell Culture}

The A549 cell line was kindly picked up from Yusuf Baran (Department of Molecular Biology and Genetics, Izmir Institute of Technology). The cells were maintained in RPMI-1640 enriched with fetal bovine serum and penicillin/ streptomycin $\left(37^{\circ} \mathrm{C}, 5 \% \mathrm{CO}_{2}\right)$.

\subsection{Corilagin Treatment}

A549 cancer cells took exposure of dimethyl sulfoxide (DMSO) vehicle (1\%) alone or increasing doses of of Corilagin $(5,10,25,50$ and $100 \mu \mathrm{M})$. 


\subsection{Cell Culture Reagents}

The medium, FBS, and penicillin/streptomycin were bought from GIBCO Life Technologies (Thermo Fisher; USA). Corilagin was purchased from Sigma Aldrich (Sigma Aldrich; Darmstadt, Germany). The WST-1 was obtained from Roche Life Sciences (Roche Life Sciences; Germany). The Caspase-3 assay was purchased from manufacturer (BioVision; USA). The JC-1 Mitochondrial Membrane Potential Detection assay was obtained from Cayman Chemical (Cayman Chemical; Ann Arbor, MI, USA).

\subsection{WST-I Assay}

The cellular impacts of Corilagin in NSCLC cells was determined by using WST-1 assay. The cells were seeded in 96-multiwell plates as a density of $1 \times 10^{4}$ cells/well. Following incubation period (24h, $48 \mathrm{~h}, 72 \mathrm{~h})$, cells were treated with increasing doses of Corilagin (5-100 $\mu \mathrm{M})$ or vehicle (DMSO, 1\%) alone as a control. Ten microliters of WST-1 solution were mixtured with cells and then incubated for $4 \mathrm{~h}$ at $37^{\circ} \mathrm{C}$. The results were read at $450 \mathrm{~nm}$ wavelength by using spectrophotometry. Cellular viability was calculated by comparison of proliferation vs. untreated cells (control, $100 \%)$.

\subsection{Caspase-3 Activity}

The colorimetric Caspase- 3 assay was to used to determine the apoptotic changes after Corilagin exposure. First, the cells were seeded in 96 -well plates $\left(5 \times 10^{5}\right.$ cells/well). After incubation period $(24 \mathrm{~h})$, cells were induced with Corilagin (50 or $100 \mu \mathrm{M}$ ) or control vehicle (DMSO, 1\%) for several time points (24-72h). After each time point, the Corilagin stimulated cells underwent lysis process by adding $50 \mu \mathrm{L}$ (chilled) and incubated for ten minutes. The supernatants were mixed with $50 \mu \mathrm{L}$ Reaction Buffer and $5 \mu \mathrm{L}$ DEVD-Pna substrate then waited two hours for incubation. The results were read at $405 \mathrm{~nm}$ wavelength by using spectrophotometry.

\subsection{The JC-I assay}

The JC-I assay was used to examine the loss of MMP after Corilagin exposure (50 or $100 \mu \mathrm{M}$ ). Briefly, the Corilagin stimulated cells $\left(5 \times 10^{5}\right.$ cells $\left./ 2 \mathrm{~mL}\right)$ were collected with centrifugation (1000 rpm, $10 \mathrm{~min}$ ). After homogenously mixing the remained pellets with $200 \mu \mathrm{L}$ medium\& $20 \mu \mathrm{L}$ JC- 1 dye, the cells were incubated for 30 minutes at $37^{\circ} \mathrm{C}$ and centrifuged. The final pellets were resuspended in 320 $\mu \mathrm{L}$ buffer and then $100 \mu \mathrm{l}$ of each sample was seeded in a 96well plate as triplicate. The results were read at Green/red $(510 \mathrm{~nm} / 585 \mathrm{~nm}$ ) wavelengths by using ELISA reader.

\subsection{Statistical Analysis}

Statistical Package for the Social Sciences software package was used to determine statistical analyzes (revision 11.5 SPSS Inc., Chicago, IL, U.S.A.). The Mann-Whitney $U$ test was used to find the mean value of results. The findings were thought statistically meanful if the $p$ value was smaller than 0.01 or 0.05 .

\section{RESULTS}

\subsection{Corilagin Suppresses A549 Cell Proliferation}

We determined that Corilagin stimulation (50 and 100 $\mu \mathrm{M})$ had statistically significant impacts in A549 cells $\left({ }^{*} p<0.05,{ }^{* *} p<0.01\right)$. IC50 values of Corilagin were calculated and found as $0.7 \mathrm{mM}$ (Figure 1).

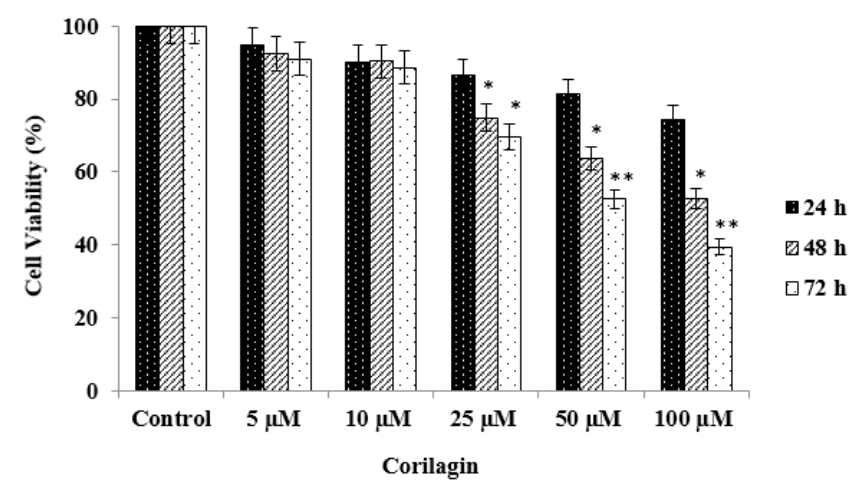

Figure 1. Effects of Corilagin on cell proliferation. WST-1 proliferation was performed using triplicate samples in three independent experiments. Statistical significance was determined using two-way analysis of variance, and $p<0.05$ was considered significant $\left(^{*} p<\right.$ $0.05 ; * *<0.01)$.

\subsection{Corilagin Improves Caspase-3 Enzyme Activity}

We found that there was a 1.5-fold increase in caspase-3 activity after Corilagin treatment ( $48 \mathrm{~h}, 50 \mu \mathrm{M})$ ). Furthermore, we also showed that that there were 2.6-fold ( $48 \mathrm{~h})$ and 2.1fold $(72 \mathrm{~h})$ changes in caspase- 3 activity for several time points after $100 \mu \mathrm{M}$ Corilagin treatment (Figure 2).

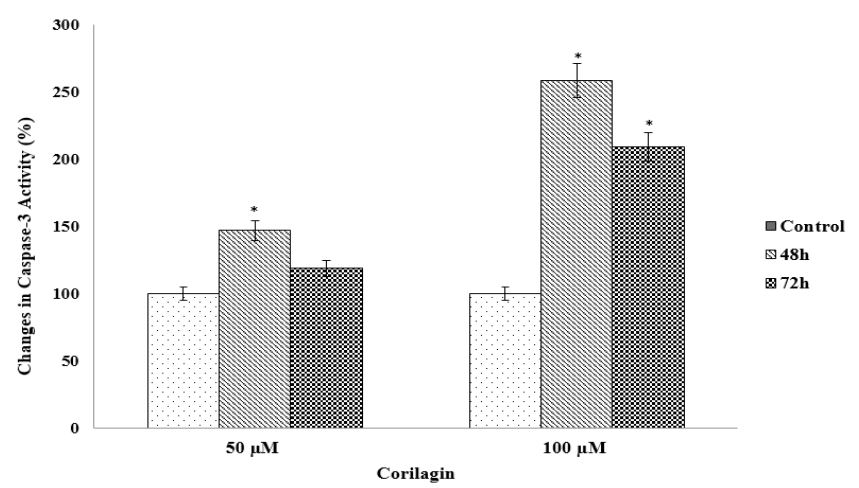

Figure 2. Effects of Corilagin on caspase-3 activity in A549 cells. Changes in caspase-3 enzyme activity in response to increasing concentrations of corilagin in A549 cells. The results are the means of two independent experiments. $p<0.05$ was considered significant 


\subsection{Corilagin Induces the Loss of Mitochondrial Membrane Potential}

The results showed that there was a 1.90-fold changes in the loss of MMP after $50 \mu \mathrm{M}$ corilagin treatment. In addition, we also found that there were 2.1-fold ( $48 \mathrm{~h}$ ) and 1.90-fold (72 h) increases in loss of MMP after $100 \mu \mathrm{M}$ Corilagin treatment (Figure 3).

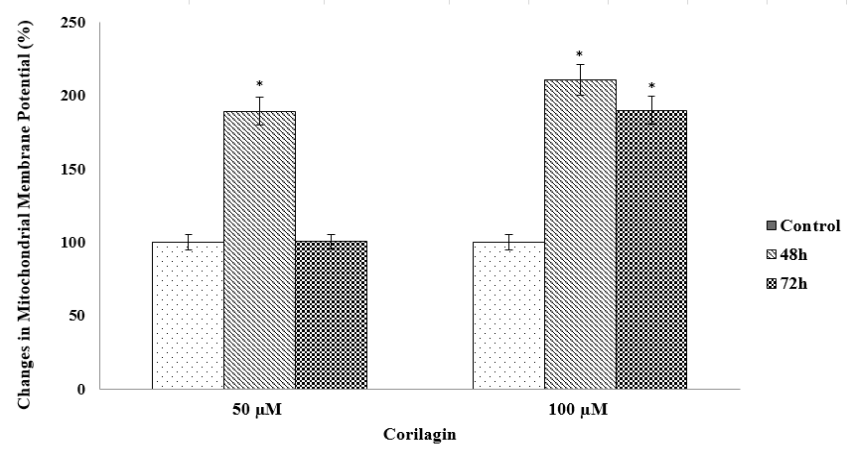

Figure 3. Effects of Corilagin on membrane potential in A549 cells. Loss of mitochondrial membrane potential (MMP) in response to increasing concentrations of corilagin in $A 549$ cells. The results are represented as the means of two independent experiments. $p<0.05$ was considered significant.

\section{DISCUSSION}

In this study, our goal was to examine antiproliferative and apoptotic effects of Corilagin in NSCLC for the first time. Herein, we identified that Corilagin could inhibit cell proliferation of A549 lung cancer cells while promoting apoptotic event like stimulation of caspase-3 activity and loss of MMP. Our finding showed correlation with previous studies. Hau and colleagues verified that Corilagin had antitumoral effects by suppressing tumoral growth in both in vivo and in vitro studies (20). They also showed that Corilagin had no side or toxic effects on liver with using alanine transaminase (ALT) and aspartate transaminase (AST) tests. Furthermore, Gambari and collegues found that combine effects of Cisplatin-Corilagin or Doxorubicin-Corilagin could sensitize Hep3B cells by increasing antitumoral effects of chemotherapeutics (21).

It had been also reported that Corilagin could cause cellular arrest at G2/M checkpoint to decrease PAKT signaling while promoting p53 gene expression in hepatocellular cancer cells (HCC) (22). Taken together, it could be concluded that Corilagin could use as a new therapeutic method for hepatocellular cancer treatment.

In our previous study, we showed that Corilagin had antiproliferative, apoptotic and genomic impacts in ovarian cancer cells. Due to results of bioinformatic analysis, we found that the phosphatidylinositol signaling system could be modulated through dose and time-dependent Corilagin stimulation. The data of Jai and collegues was also supportive for our findings (23). According to their study, Corilagin could stimulate G2/M cell arrest in ovarian cancer cells in line with studies in HCC. Moreover, they demonstrated that Corilagin could directly inhibit TGF-B secretion to suppress activation of SMAD and ERK/AKT signaling pathways.

There were only two studies which had investigated the reparative impacts of Corilagin exposure in experimental models. The first study was about determination the behavior of Corilagin against tobacco consumption (24). Muresan and collegues discovered that Corilagin could reverse cellular disruption between intercellular junctions through promoting expression of connexin proteins in lung epithelial cells (24). In the second study, Wang and collegues examined the impacts of Corilagin in experimental pulmonary fibrosis model. They showed that Corilagin could repair damages in lung epithelial cells by stimulating cytokine secretion and TGF-B activation (25).

\section{CONCLUSION}

In conclusion, we suggested that Corilagin has potential anticarcinogenic properties to use as a new therapeutic on NSCLC treatment. Further studies will help us to understand the molecular mechanism underlying corilagin stimulation in cancer models.

Financial Disclosure: This work was funded by Istanbul University Scientific Research Project Number: 29026.

\section{REFERENCES}

[1] Pao W, Girard N. New driver mutations in non-small-cell lung cancer. Lancet Oncol 2011;12:175-180.

[2] Travis WD. Classification of lung cancer. Semin Roentgenol 2011;46:178-186.

[3] Wu Q, Chen YF, Fu J., You QH, Wang SH, Huang X, Feng XJ, Zhang $\mathrm{SH}$. Short hairpin RNA-mediated down-regulation of CENPA attenuates the aggressive phenotype of lung adenocarcinoma cells. Cell Oncol 2014;37:399-407.

[4] Koren A., Motaln H., Cufer T. Lung cancer stem cells: a biological and clinical perspective. Cell Oncol 2013;36: 265-275.

[5] Peled N, Wynes MW, Ikeda N, Ohira T, Yoshida K, Qian J, Illoze $M$, Brenner R, Kato $Y$, Mascaux C, Hirsch FR. Insulin-like growth factor-1 receptor (IGF-1R) as a biomarker for resistance to the tyrosine kinase inhibitor gefitinib in non-small cell lung cancer. Cell Oncol 2013;36:277-288.

[6] Maier A, Peille AL, Vuaroqueaux V, Lahn M. Anti-tumor activity of the TGF- $\beta$ receptor kinase inhibitor galunisertib (LY2157299 monohydrate) in patient-derived tumor xenografts. Cell Oncol 2015; 38: 131-144.

[7] Ulivi P, Silvestrini R. Role of quantitative and qualitative characteristics of free circulating DNA in the management of patients with non-small cell lung cancer. Cell Oncol 2013;36:439-448.

[8] Giaccone G. Twenty-five years of treating advanced NSCLC: what have we achieved? Ann Oncol 2004;4:81-83.

[9] Neal JW, Sequist LV. Exciting new targets in lung cancer therapy: ALK, IGF-1R, HDAC, and Hh. Curr Treat Options in Oncol 2010;11:36-44. 
[10] Sangha R, Lara PN, Mack PC, Gandara DR. Beyond antiepidermal growth factor receptors and antiangiogenesis strategies for nonsmall cell lung cancer: exploring a new frontier. Curr Opin Oncol 2009;21:116-123.

[11] Mateen S, Raina K, Agarwal R. Chemopreventive and anticancer efficacy of silibinin against growth and progression of lung cancer. Nutr Cancer J 2013;65:3-11.

[12] Hau DK, Gambari R, Wong RS, Yuen MC, Cheng GY, Tong CS, Zhu GY, Leung AK, Lai PB, Lau FY, Chan AK, Wong WY, Kok $\mathrm{SH}$, Cheng CH, Kan CW, Chan AS, Chui CH, Tang JC, Fong DW. Phyllanthus urinaria extract attenuates acetamionophen induced hepatotoxicity: involvement of cytochrome P450 CYP2E1. Phytomedicine 2009;16:751- 760.

[13] Shen ZQ, Dong ZJ, Peng H, Liu JK. Modulation of PAI-1 and tPA activity and thrombolytic effects of corilagin. Planta Med 2003;69:1109-1112.

[14] Sudjaroen Y, Hull WE, Erben G, Wuertele GM, Changbumrung $S$, Ulrich CM, Owen RW. Isolation and characterization of ellagitannins as the major polyphenolic components of Longan (Dimocarpus longan Lour) seeds. Phytochemistry 2012;77:226-237.

[15] Okabe S, Suganuma M, Imayoshi Y, Taniguchi S, Yoshida T, Fujiki H. New TNF-a releasing inhibitors, geraniin and corilagin, in leaves of acer nikoense, megusurino-ki. Biological and Pharmaceutical Bulletin 2001;24,10:1145-1148.

[16] Duan W, Yu Y, Zhang L. Antiatherogenic effects of phyllanthus emblica associated with corilagin and its analogue. Yakugaku Zasshi 2005;125:587-591.

[17] Cheng JT, Lin TC, Hsu FL. Antihypertensive effect of corilagin in the rat. Can. J Physiol Pharmacol 1995;73:1425-1429.
[18] Duan WG, Shen ZQ, Yan M, Yun Y, Zhang LY. Corilagin, a promis-ing natural product to treat cardiovascular diseases. In: Singh VK, Mishra SK, Govil JN, editors. Phytopharmacology and Therapeutic Values II. Houston: Studium Press; 2008:163172.

[19] Kinoshita S, Inoue Y, Nakama S, Ichiba T, Aniya Y. Antioxidant and hepatoprotective actions of medicinal herb, Terminalia catappa L. from Okinawa Island and its tannin corilagin. Phytomedicine 2007;14, 11: 755-762.

[20] Hau DK, Zhu GY, Leung AK, Wong RS, Cheng GY, Lai PB, Tong SW, Lau FY, Chan KW, Wong WY, Lam KH, Cheng CH, Cheung F, Chui $\mathrm{CH}$, Gambari R, Fong DW. In vivo anti-tumour activity of corilagin on Нер3В hepatocellular carcinoma. Phytomedicine 2010;15, 18(1):11-15.

[21] Gambari R, Hau DK, Wong WY, Chui CH. Sensitization of Hep3B hepatoma cells to cisplatin and doxorubicin by corilagin. Phytother Res 2014;28(5):781-783.

[22] Ming Y, Zheng Z, Chen L, Zheng G, Liu S, Yu Y, Tong Q. Corilagin inhibits hepatocellular carcinoma cell proliferation by inducing G2/M phase arrest. Cell Biol Int 2013;37(10):1046-1054.

[23] Jia L, Jin H, Zhou J, Chen L, Lu Y, Ming Y, Yu Y. A potential antitumor herbal medicine, Corilagin, inhibits ovarian cancer cell growth through blocking the TGF- $\beta$ signaling pathways. BMC Complement Altern Med 2013;15: 13:33.

[24] Muresan XM, Cervellati F, Sticozzi C, Belmonte G, Chui CH, Lampronti I, Borgatti M, Gambari R, Valacchi G. The loss of cellular junctions in epithelial lung cells induced by cigarette smoke is attenuated by corilagin. Oxid Med Cell Longev 2015;2015: 631-758.

[25] Wang Z, Guo QY, Zhang XJ, Li X, Li WT, Ma XT, Ma LJ. Corilagin attenuates aerosol bleomycin-induced experimental lung injury. Int J Mol Sci 2014;30:15(6):9762-9779. 